\title{
On a successive property of strongly starlikeness for multivalent functions
}

\section{Mamoru Nunokawa ${ }^{1}$ - Janusz Sokół ${ }^{2}$}

Received: 31 May 2019 / Accepted: 6 August 2019 / Published online: 31 August 2019

(c) The Author(s) 2019

\section{Abstract}

For $f$ analytic in the unit disk $\mathbb{D}$, of the form $f(z)=z^{p}+\cdots$, we consider some consequences of strongly starlikeness of $f^{(p-1)}(z) / p$ !.

Keywords Starlike $\cdot$ Strongly starlike $\cdot$ Multivalent

Mathematics Subject Classification Primary 30C45; Secondary 30C80

\section{Introduction}

We denote by $\mathcal{H}$ the class of functions $f(z)$ which are holomorphic in the open unit disc $\mathbb{D}=\{z \in \mathbb{C}:|z|<1\}$. Denote by $\mathcal{A}_{p}, p \in \mathbb{N}=\{1,2, \ldots\}$, the class of functions $f(z) \in \mathcal{H}$ given by

$$
f(z)=z^{p}+\sum_{n=p+1}^{\infty} a_{n} z^{n}, \quad(z \in \mathbb{D}) .
$$

Lemma 1.1 [2, Theorem 5] If $f(z) \in \mathcal{A}_{p}$, then for all $z \in \mathbb{D}$, we have

$$
\mathfrak{R e}\left\{\frac{z f^{(p)}(z)}{f^{(p-1)}(z)}\right\}>0 \Rightarrow \forall k \in\{1, \ldots, p-1\}: \quad \mathfrak{R e}\left\{\frac{z f^{(k)}(z)}{f^{(k-1)}(z)}\right\}>0 .
$$

In this paper we consider a generalization of the above result. In Lemma 1.1 we have assumed that $z f^{(p)}(z) / f^{(p-1)}(z)$ lies in the right half-plane while in this paper we work with a sector. The problem we solve here is: for what values of $\alpha, \beta$ does an analytic function of the form (1.1) satisfy

Janusz Sokół

jsokol@ur.edu.pl

Mamoru Nunokawa

mamoru_nuno@doctor.nifty.jp

1 University of Gunma, Hoshikuki-cho 798-8, Chuou-Ward, Chiba 260-0808, Japan

2 Faculty of Mathematics and Natural Sciences, University of Rzeszów, ul. Prof. Pigonia 1, 35-310

Rzeszow, Poland 


$$
\left|\arg \left\{\frac{z f^{(p)}(z)}{f^{(p-1)}(z)}\right\}\right|<\frac{\pi \alpha}{2} \Rightarrow \forall k \in\{1, \ldots, p-1\}:\left|\arg \left\{\frac{z f^{(k)}(z)}{f^{(k-1)}(z)}\right\}\right|<\frac{\pi \beta}{2} ?
$$

Recall that if $f(z) \in \mathcal{A}_{p}$ and

$$
\mathfrak{R e}\left\{\frac{z f^{(p)}(z)}{f^{(p-1)}(z)}\right\}>0 \quad(z \in \mathbb{D})
$$

then $f^{(p-1)}(z) / p ! \in \mathcal{A}_{1}$ is univalent in $\mathbb{D}$ and $f^{(p-1)}(z) / p$ ! is called a starlike function. If $f(z) \in \mathcal{A}_{p}, \gamma \in(0,1]$, and

$$
\arg \left|\frac{z f^{(p)}(z)}{f^{(p-1)}(z)}\right|<\frac{\pi \gamma}{2}, \quad z \in \mathbb{D}
$$

then $f^{(p-1)}(z) / p$ ! is called a strongly starlike function of order $\gamma$ and such functions we consider in the paper. This class for the case $p=1$ was introduced by Brannan and Kirwan [1]. Also, if $f(z) \in \mathcal{A}_{p}$ satisfies (1.3), then $f(z)$ is called $p$-valently strongly starlike function of order $\gamma$. For the proof of main result we need the following lemma.

Lemma 1.2 [3] Let $q(z)=1+\sum_{n \geq m}^{\infty} c_{n} z^{n}, c_{m} \neq 0$ be analytic function in $|z|<1$ with $q(0)=1, q(z) \neq 0$. If there exists a point $z_{0},\left|z_{0}\right|<1$, such that

$$
|\arg \{q(z)\}|<\frac{\pi \beta}{2} \text { for }|z|<\left|z_{0}\right|
$$

and

$$
\left|\arg \left\{q\left(z_{0}\right)\right\}\right|=\frac{\pi \beta}{2}
$$

for some $\beta>0$, then we have

$$
\frac{z_{0} q^{\prime}\left(z_{0}\right)}{q\left(z_{0}\right)}=\frac{2 i k \arg \left\{q\left(z_{0}\right)\right\}}{\pi}
$$

for some $k \geq m\left(a+a^{-1}\right) / 2 \geq m$, where

$$
\left\{q\left(z_{0}\right)\right\}^{1 / \beta}= \pm i a, \text { and } a>0 .
$$

\section{Main results}

For given $0<\beta_{s-1} \leq 1$ let us consider the number

$$
\beta_{s}=\beta_{s-1}+\frac{2}{\pi} \tan ^{-1} \frac{\beta_{s-1} n\left(\beta_{s-1}\right) \sin \left[\pi\left(1-\beta_{s-1}\right) / 2\right]}{\operatorname{sm}\left(\beta_{s-1}\right)+\beta_{s-1} n\left(\beta_{s-1}\right) \cos \left[\pi\left(1-\beta_{s-1}\right) / 2\right]}, \quad s=2,3, \ldots, p,
$$

where

$$
m\left(\beta_{s-1}\right)=\left(1+\beta_{s-1}\right)^{\left(1+\beta_{s-1}\right) / 2}, \text { and } n\left(\beta_{s-1}\right)=\left(1-\beta_{s-1}\right)^{\left(1-\beta_{s-1}\right) / 2} .
$$


Notice that if $0<\beta_{s-1} \leq 1$, then $0<\beta_{s} \leq 1$ too because from (2.1), (2.2), we have

$$
\begin{aligned}
\beta_{s} & =\beta_{s-1}+\frac{2}{\pi} \tan ^{-1} \frac{\beta_{s-1} n\left(\beta_{s-1}\right) \sin \left[\pi\left(1-\beta_{s-1}\right) / 2\right]}{\operatorname{sm}\left(\beta_{s-1}\right)+\beta_{s-1} n\left(\beta_{s-1}\right) \cos \left[\pi\left(1-\beta_{s-1}\right) / 2\right],} \\
& \leq \beta_{s-1}+\frac{2}{\pi} \tan ^{-1} \frac{\beta_{s-1} n\left(\beta_{s-1}\right) \sin \left[\pi\left(1-\beta_{s-1}\right) / 2\right]}{\beta_{s-1} n\left(\beta_{s-1}\right) \cos \left[\pi\left(1-\beta_{s-1}\right) / 2\right],} \\
& =1 .
\end{aligned}
$$

Therefore, if we have a number $\beta_{1} \in(0,1]$, then from $(2.1)$, we can find a sequence $\beta_{p}, \beta_{p-1}, \ldots, \beta_{2}, \beta_{1}$, such that

$$
0<\beta_{1} \leq \beta_{2} \leq \cdots \leq \beta_{p-1} \leq \beta_{p} \leq 1
$$

Theorem 2.1 Let $f(z) \in \mathcal{A}_{p}, p \geq 2$. For given $\beta_{p-1} \in(0,1]$ there exists $\beta_{p} \in(0,1]$ of the form (2.1) such that for all $z \in \mathbb{D}$, we have

$$
\left|\arg \left\{\frac{z f^{(p)}(z)}{f^{(p-1)}(z)}\right\}\right|<\frac{\pi \beta_{p}}{2} \Rightarrow\left|\arg \left\{\frac{z f^{(p-1)}(z)}{f^{(p-2)}(z)}\right\}\right|<\frac{\pi \beta_{p-1}}{2} .
$$

Proof Let us put

$$
q_{1}(z)=\frac{z f^{(p-1)}(z)}{2 f^{(p-2)} z}, \quad q_{1}(0)=1
$$

Then it follows that

$$
\frac{z q_{1}^{\prime}(z)}{q_{1}(z)}=1+\frac{z f^{(p)}(z)}{f^{(p-1)}(z)}-\frac{z f^{(p-1)}(z)}{f^{(p-2)}(z)}
$$

and

$$
2 q_{1}(z)+\frac{z q_{1}^{\prime}(z)}{q_{1}(z)}=1+\frac{z f^{(p)}(z)}{f^{(p-1)}(z)}
$$

and so

$$
\arg \left\{q_{1}(z)\right\}+\arg \left\{2+\frac{z q_{1}^{\prime}(z)}{q_{1}^{2}(z)}\right\}=\arg \left\{1+\frac{z f^{(p)}(z)}{f^{(p-1)}(z)}\right\} .
$$

If there exists a point $z_{0} \in \mathbb{D}$ such that

$$
\begin{aligned}
& \left|\arg \left\{q_{1}(z)\right\}\right|<\pi \beta_{p-1} / 2 \text { for }|z|<\left|z_{0}\right|, \quad\left|\arg \left\{q_{1}\left(z_{0}\right)\right\}\right|=\pi \beta_{p-1} / 2 \\
& \left\{q_{1}\left(z_{0}\right)\right\}^{1 / \beta_{p-1}}= \pm i a, \quad \text { and } a>0
\end{aligned}
$$

then from Lemma 1.2, we have

$$
\frac{z_{0} q_{1}^{\prime}\left(z_{0}\right)}{q_{1}\left(z_{0}\right)}=\frac{2 i k \arg \left\{q_{1}\left(z_{0}\right)\right\}}{\pi}
$$

for some real $k$ with $k \geq\left(a+a^{-1}\right) / 2 \geq 1$. For the case $\arg \left\{q_{1}\left(z_{0}\right)\right\}=\pi \beta_{p-1} / 2$, we have

$$
\begin{aligned}
\arg \left\{\frac{z_{0} f^{(p)}\left(z_{0}\right)}{f^{(p-1)}\left(z_{0}\right)}\right\} & \geq \arg \left\{1+\frac{z_{0} f^{(p)}\left(z_{0}\right)}{f^{(p-1)}\left(z_{0}\right)}\right\} \\
& =\arg \left\{q_{1}\left(z_{0}\right)\right\}+\arg \left\{2+\frac{z_{0} q_{1}^{\prime}\left(z_{0}\right)}{q_{1}\left(z_{0}\right)} \frac{1}{q_{1}\left(z_{0}\right)}\right\} \\
& \geq \frac{\pi \beta_{p-1}}{2}+\arg \left\{2+e^{i \pi\left(1-\beta_{p-1}\right) / 2} \frac{1}{(i a)^{\beta_{p-1}}}\right\},
\end{aligned}
$$


where $\left\{q_{1}\left(z_{0}\right)\right\}^{1 / \beta_{p-1}}=i a$ and $a$ is a positive real number. Applying Lemma 1.2 we obtain

$$
\begin{aligned}
\arg & \left\{\frac{z_{0} f^{(p)}\left(z_{0}\right)}{f^{(p-1)}\left(z_{0}\right)}\right\} \\
\geq & \frac{\pi \beta_{p-1}}{2}+\arg \left\{e ^ { i \pi ( 1 - \beta _ { p - 1 } ) / 2 } \left(\left(\frac{1+\beta_{p-1}}{1-\beta_{p-1}}\right)^{\left(1-\beta_{p-1}\right) / 2}\right.\right. \\
& \left.\left.+\left(\frac{1+\beta_{p-1}}{1-\beta_{p-1}}\right)^{-\left(1+\beta_{p-1}\right) / 2}\right)+2\right\} \\
= & \frac{\pi \beta_{p-1}}{2}+\tan ^{-1} \frac{\frac{\beta_{p-1}}{1-\beta_{p-1}}\left(\frac{1-\beta_{p-1}}{1+\beta_{p-1}}\right)^{\left(1+\beta_{p-1}\right) / 2} \sin \frac{\pi\left(1-\beta_{p-1}\right)}{2}}{2+\frac{\beta_{p-1}}{1-\beta_{p-1}}\left(\frac{1-\beta_{p-1}}{1+\beta_{p-1}}\right)^{\left(1+\beta_{p-1}\right) / 2} \cos \frac{\pi\left(1-\beta_{p-1}\right)}{2}} \\
= & \frac{\pi \beta_{p-1}}{2}+\tan ^{-1} \frac{\beta_{p-1} n\left(\beta_{p-1}\right) \sin \frac{\pi\left(1-\beta_{p-1}\right)}{2}}{2 m\left(\beta_{p-1}\right)+\beta_{p-1} n\left(\beta_{p-1}\right) \cos \frac{\pi\left(1-\beta_{p-1}\right)}{2}} .
\end{aligned}
$$

From (2.1), we can see that

$$
\arg \left\{\frac{z_{0} f^{(p)}\left(z_{0}\right)}{f^{(p-1)}\left(z_{0}\right)}\right\} \geq \frac{\pi \beta_{p}}{2} .
$$

This contradicts hypothesis in (2.4).

For the case $\arg \left\{q_{1}\left(z_{0}\right)\right\}=-\pi \beta_{p-1} / 2$, applying the same method as the above, gives

$$
\arg \left\{\frac{z_{0} f^{(p)}\left(z_{0}\right)}{f^{(p-1)}\left(z_{0}\right)}\right\} \leq-\frac{\pi \beta_{p}}{2} .
$$

This also contradicts hypothesis in (2.4) and therefore, we have

$$
\left|\arg \left\{q_{1}(z)\right\}\right|<\pi \beta_{p-1} / 2 \text { for }|z|<|1| .
$$

This completes the proof.

Let us go to next step and define the function

$$
q_{2}(z)=\frac{z f^{(p-2)}(z)}{3 f^{(p-3)}(z)}, \quad q_{2}(0)=1
$$

and applying the same method as the above, we have the following theorem.

Theorem 2.2 Let $f(z) \in \mathcal{A}_{p}, p \geq 2,0<\beta_{2} \leq 1$ and suppose that

$$
\left|\arg \left\{\frac{z f^{(p)}(z)}{f^{(p-1)}(z)}\right\}\right|<\frac{\pi \beta_{p}}{2}, \quad z \in \mathbb{D} .
$$

Then we have

$$
\left|\arg \left\{\frac{z f^{(p-2)}(z)}{f^{(p-3)}(z)}\right\}\right|<\frac{\pi \beta_{p-2}}{2}, \quad z \in \mathbb{D} .
$$

where $\beta_{p-2}$ we obtain from $\beta_{p-1}$ using formula (2.1). Furthermore, 


$$
\left|\arg \left\{\frac{z f^{(p-1)}(z)}{f^{(p-2)}(z)}\right\}\right|<\frac{\pi \beta_{p-1}}{2}, \quad z \in \mathbb{D},
$$

and where $\beta_{p-1}$ we obtain from $\beta_{p}$ using formula (2.1) too.

Applying the same step as the above and under the hypothesis of Theorem 2.1, we have the following theorem

Theorem 2.3 Let $f(z) \in \mathcal{A}_{p}, p \geq 2$. For given $\beta_{1} \in(0,1]$ there exist $\beta_{k} \in(0,1], k=$ $2, \ldots, p$, of the form (2.1) such that for all $z \in \mathbb{D}$, we have

$$
\left|\arg \left\{\frac{z f^{(p)}(z)}{f^{(p-1)}(z)}\right\}\right|<\frac{\pi \beta_{p}}{2} \Rightarrow \forall k \in\{1, \ldots, p-1\}: \quad \arg \left\{\frac{z f^{(k)}(z)}{f^{(k-1)}(z)}\right\} \mid<\frac{\pi \beta_{k}}{2} .
$$

Furthermore

$$
0<\beta_{1} \leq \beta_{2} \leq \cdots \leq \beta_{p-1} \leq \beta_{p} \leq 1 .
$$

It is easy to see that Theorem 2.3 holds for the case $\beta_{p}=\beta_{p-1}=\cdots=\beta_{1}=1$ and then Theorem 2.3 becomes Lemma 1.1 and in this sense Theorem 2.3 improves Lemma 1.1.

Corollary 2.4 Let $f(z) \in \mathcal{A}_{p}, p \geq 2$. If $\beta_{1} \in(0,1]$ and $\beta_{k} \in(0,1], k=2, \ldots, p$ are of the form (2.1), then for all $k=1, \ldots, p-1$ and for all $z \in \mathbb{D}$, we have

$$
\left|\arg \left\{\frac{z f^{(p)}(z)}{f^{(p-1)}(z)}\right\}\right|<\frac{\pi \beta_{k}}{2} \Rightarrow\left|\arg \left\{\frac{z f^{(k)}(z)}{f^{(k-1)}(z)}\right\}\right|<\frac{\pi \beta_{k}}{2} .
$$

Proof For given $\beta_{1} \in(0,1]$ there exist $\beta_{k} \in(0,1], k=2, \ldots, p$, of the form $(2.1)$ such that

$$
\left|\arg \left\{\frac{z f^{(p)}(z)}{f^{(p-1)}(z)}\right\}\right|<\frac{\pi \beta_{p}}{2} \Rightarrow \forall k \in\{1, \ldots, p-1\}:\left|\arg \left\{\frac{z f^{(k)}(z)}{f^{(k-1)}(z)}\right\}\right|<\frac{\pi \beta_{k}}{2} .
$$

where

$$
0<\beta_{1} \leq \beta_{2} \leq \cdots \leq \beta_{p-1} \leq \beta_{p} \leq 1 .
$$

Therefore, from (2.11), we have

$$
\begin{aligned}
& \left|\arg \left\{\frac{z f^{(p)}(z)}{f^{(p-1)}(z)}\right\}\right|<\frac{\pi \beta_{k}}{2} \Rightarrow\left|\arg \left\{\frac{z f^{(p)}(z)}{f^{(p-1)}(z)}\right\}\right|<\frac{\pi \beta_{p}}{2} \\
& \Rightarrow\left|\arg \left\{\frac{z f^{(k)}(z)}{f^{(k-1)}(z)}\right\}\right|<\frac{\pi \beta_{k}}{2} .
\end{aligned}
$$

Corollary 2.5 If $f(z) \in \mathcal{A}_{p}, p \geq 2$, then for all $\gamma \in(0,1]$ and for all $k \in\{1, \ldots, p\}$ and for all $s \in\{k, \ldots, p-1\}$, and for all $z \in \mathbb{D}$, we have

$$
\left|\arg \left\{\frac{z f^{(s)}(z)}{f^{(s-1)}(z)}\right\}\right|<\frac{\pi \gamma}{2} \Rightarrow\left|\arg \left\{\frac{z f^{(k)}(z)}{f^{(k-1)}(z)}\right\}\right|<\frac{\pi \gamma}{2} .
$$

From the properties of the sequence (2.1) we have following corollary. 
Corollary 2.6 Let $f(z) \in \mathcal{A}_{p}, p \geq 2,0<\beta_{p} \leq 1$ and suppose that

$$
\left|\arg \left\{\frac{z f^{(p)}(z)}{f^{(p-1)}(z)}\right\}\right|<\frac{\pi \beta_{p}}{2}, \quad z \in \mathbb{D} \text {. }
$$

Then we have

$$
\begin{aligned}
\sup _{z \in \mathbb{D}}\left|\arg \left\{\frac{z f^{(p)}(z)}{f^{(p-1)}(z)}\right\}\right| & >\sup _{z \in \mathbb{D}}\left|\arg \left\{\frac{z f^{(p-1)}(z)}{f^{(p-2)}(z)}\right\}\right|>\cdots>\sup _{z \in \mathbb{D}}\left|\arg \left\{\frac{z f^{(k)}(z)}{f^{(k-1)}(z)}\right\}\right| \\
& >\sup _{z \in \mathbb{D}}\left|\arg \left\{\frac{z f^{(k-1)}(z)}{f^{(k-2)}(z)}\right\}\right|>\cdots>\sup _{z \in \mathbb{D}}\left|\arg \left\{\frac{z f^{\prime}(z)}{f(z)}\right\}\right| .
\end{aligned}
$$

Theorem 2.7 Let $\beta=\alpha+(2 / \pi) \tan ^{-1} \alpha$ and $f(z) \in \mathcal{A}_{p}, p \geq 2$. Suppose also that

$$
\left|\arg \left\{f^{(p)}(z)\right\}\right|<\frac{\pi \beta}{2}, \quad z \in \mathbb{D} \text {. }
$$

Then we have

$$
\left|\arg \left\{\frac{z f^{\prime}(z)}{f(z)}\right\}\right|<\frac{\pi \beta_{1}}{2}, \quad z \in \mathbb{D},
$$

where $\beta_{1}$ is described in (2.1) with $\beta_{p}=\alpha+\beta$.

Proof If

$$
\left|\arg \left\{\frac{f^{(p-1)}(z)}{z}\right\}\right|<\frac{\pi \alpha}{2}
$$

in $|z<| z_{0} \mid$ and

$$
\arg \left\{\frac{f^{(p-1)}\left(z_{0}\right)}{z_{0}}\right\}=\frac{\pi \alpha}{2} \quad \text { or } \quad \arg \left\{\frac{f^{(p-1)}\left(z_{0}\right)}{z_{0}}\right\}=-\frac{\pi \alpha}{2},
$$

then for the first case in (2.15), from Lemma 1.2, we have

$$
\frac{z_{0} f^{(p)}\left(z_{0}\right)}{f^{(p-1)}\left(z_{0}\right)}-1=i k \alpha
$$

for some $k \geq 1$, This gives

$$
\begin{aligned}
\arg \left\{f^{(p)}\left(z_{0}\right)\right\} & =\arg \left\{\frac{f^{(p-1)}\left(z_{0}\right)}{z_{0}}(i k \alpha+1)\right\} \\
& =\arg \left\{\frac{f^{(p-1)}\left(z_{0}\right)}{z_{0}}\right\}+\arg \{i k \alpha+1\} \\
& \geq \frac{\pi}{2}\left\{\alpha+(2 / \pi) \tan ^{-1} \alpha\right\}=-\frac{\pi \beta}{2}
\end{aligned}
$$

This contradicts hypothesis (2.12). In the second case in (2.15), applying the same method as in the first case, we obtain

$$
\arg \left\{f^{(p)}\left(z_{0}\right)\right\} \leq-\frac{\pi}{2}\left\{\alpha+(2 / \pi) \tan ^{-1} \alpha\right\}=\frac{\pi \beta}{2} .
$$


This also contradicts hypothesis (2.12). So (2.14) holds in the whole unit disc $\mathbb{D}$. From (2.12) and (2.14), we have

$$
\begin{aligned}
\left|\arg \left\{\frac{z f^{(p)}(z)}{f^{(p-1)}(z)}\right\}\right| & =\left|\arg \left\{f^{(p)}(z) \frac{z}{f^{(p-1)}(z)}\right\}\right| \\
& \leq\left|\arg \left\{f^{(p)}(z)\right\}\right|+\left|\arg \left\{\frac{f^{(p-1)}(z)}{z}\right\}\right| \\
& <\frac{\pi(\alpha+\beta)}{2}, \quad z \in \mathbb{D} .
\end{aligned}
$$

Applying Theorem 2.3, we obtain (2.13).

Open Access This article is distributed under the terms of the Creative Commons Attribution 4.0 International License (http://creativecommons.org/licenses/by/4.0/), which permits unrestricted use, distribution, and reproduction in any medium, provided you give appropriate credit to the original author(s) and the source, provide a link to the Creative Commons license, and indicate if changes were made.

\section{References}

1. Brannan, D.A., Kirwan, W.E.: On some class of bounded univalent functions. J. Lond. Math. Soc. 2(1), 1431-1443 (1969)

2. Nunokawa, M.: On the theory of multivalent functions. Tsukuba J. Math. 11(2), 273-286 (1987)

3. Nunokawa, M.: On the order of strongly starlikeness of strongly convex functions. Proc. Jpn. Acad. Ser. A 69(7), 234-237 (1993)

Publisher's Note Springer Nature remains neutral with regard to jurisdictional claims in published maps and institutional affiliations. 